\title{
TAMS COMPANIONS OF CLASSICAL CEPHEIDS
}

\author{
NANCY REMAGE EVANS \\ The Institute of Space and Terrestrial Science \\ 4850 Keele St \\ North York, M3J 3K1, Ontario, Canada
}

IUE low resolution spectra are an excellent way to determine the temperatures of the hot companions of binary Cepheids. The spectral types of the companions are derived by comparing the spectra with the spectra of standard stars. Absolute magnitudes are calculated from the magnitude difference between the two stars and the absolute magnitude of the Cepheid. In this study, eight binaries containing a Cepheid and a hot companion evolved beyond the ZAMS are discussed.

The binary components are compared with isochrones using several values of main sequence core convective overshoot: Stothers and Chin (1991): no convective overshoot, Schaller, et al. (1992): moderate convective overshoot, and Bertelli, et al. (1986): full convective overshoot. Half the systems are well matched by evolutionary tracks or isochrones with little or no convective overshoot. Four of the systems, however, cannot be matched by any current isochrone (with any overshoot value), in the sense that the companions are too cool for the isochrone appropriate to the Cepheid. Figure 1 shows the theoretical HR diagram for the sample with the four "renegade" systems identified (KN Cen, RW Cam, SV Per, and AW Per), compared with the evolutionary tracks of the Geneva group (Schaller, et al, 1992). Even for the full overshoot models, the mass difference between the two stars is too large to be consistent with companions at the TAMS (Evans, 1993).

Furthermore, these systems occur throughout the whole Cepheid period or mass range, indicating that the cause is not a mass dependent parameter. Rotation is a possible explanation.

This research was supported by a Natural Sciences and Engineering Research Council (NSERC), Canada to NRE.

\section{References}

Bertelli, G., Bressan, A., Chiosi, C., and Angerer, K.: 1986, Astronomy and Astrophysics, Supplement Series 66, 191

Evans, N. R. 1993 Astrophysical Journal, submitted

Schaller, G. Schaerer, D., Meynet, G., and Maeder, A.: 1992, Astronomy and Astrophysics, Supplement Series 96, 269

Stothers, R. B. and Chin: 1991, Astrophysical Journal 381, L67 


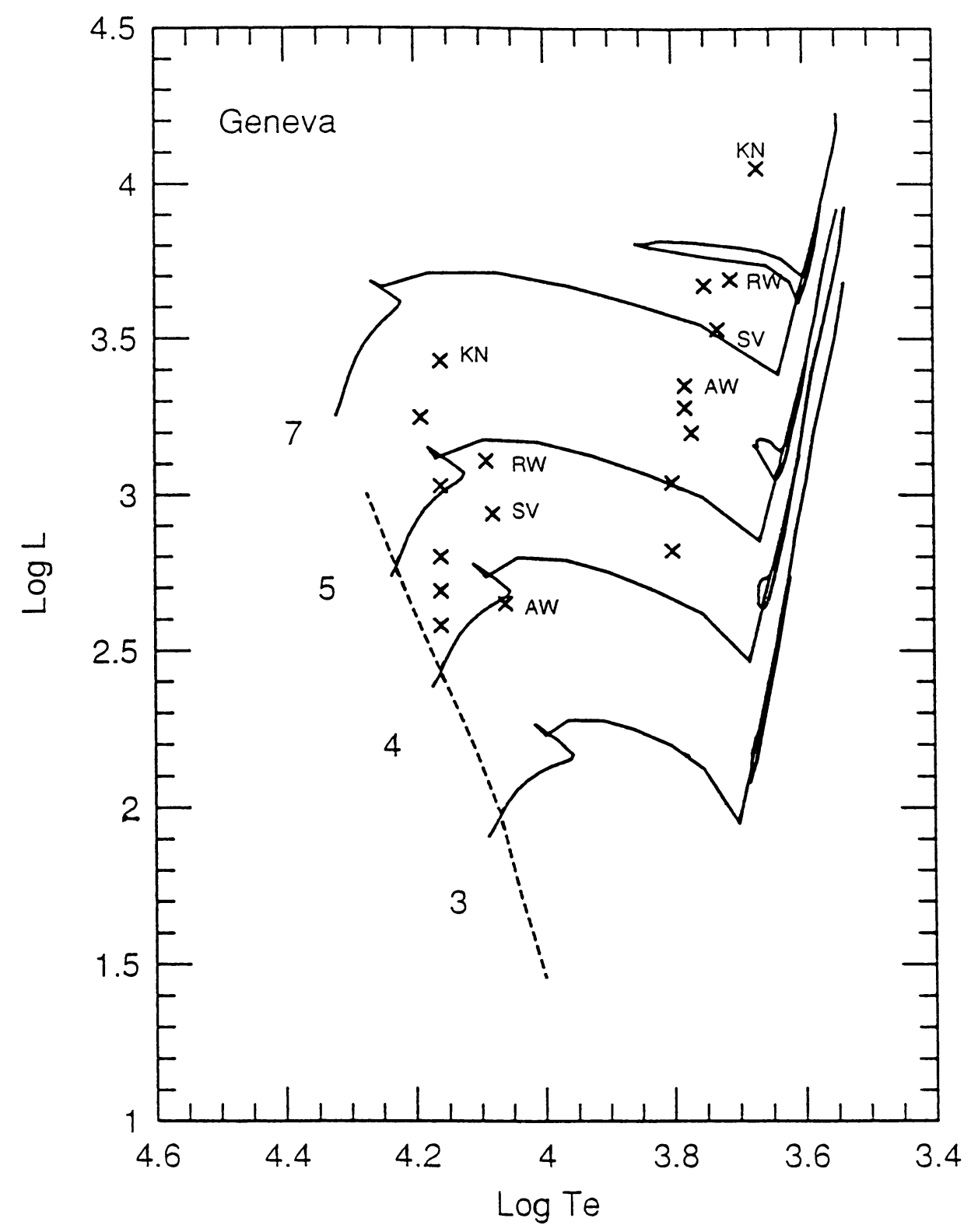

Figure 1. Binary systems containing a Cepheid and a hot companion evolved beyond the ZAMS compared with evolutionary tracks with moderate convective overshoot. The components of the four systems with surprisingly cool companions are identinied. Note that in all four cases the mass difference between the two components is too large to be consistent with companions at the very end of their main sequence lifetimes. 